ROM2F-96/23

September 9, 2018

\title{
Open Descendants in Conformal Field Theory
}

\author{
Augusto Sagnotti and Yassen S. Stanev Đ \\ Dipartimento di Fisica \\ Università di Roma "Tor Vergata" \\ I.N.F.N. - Sezione di Roma "Tor Vergata" \\ Via della Ricerca Scientifica, 100133 Roma ITALY
}

\begin{abstract}
Open descendants extend Conformal Field Theory to unoriented surfaces with boundaries. The construction rests on two types of generalizations of the fusion algebra. The first is needed even in the relatively simple case of diagonal models. It leads to a new tensor that satisfies the fusion algebra, but whose entries are signed integers. The second is needed when dealing with non-diagonal models, where Cardy's ansatz does not apply. It leads to a new tensor with positive integer entries, that satisfies a set of polynomial equations and encodes the classification of the allowed boundary operators.
\end{abstract}

Based on Talks Presented by A. Sagnotti at the CERN Meeting on STU Dualities, Dec. 1995, and by Ya.S. Stanev at the 1996 Rome Triangle Meeting, March 1996.

\footnotetext{
${ }^{1}$ I.N.F.N. Fellow, on Leave from Institute for Nuclear Research and Nuclear Energy, Bulgarian Academy of Sciences, BG-1784 Sofia, BULGARIA.
} 


\section{Introduction}

A byproduct of String Duality is a renewed interest in open-string theories [1]. These [2] provide also a relatively simple instance of the interplay between different dimensions, an emerging aspect of Field Theory that is rapidly affecting long-held views on such fundamental issues as chirality and anomalies [3]. We have decided to depart slightly from the CERN talk, a general survey on open-string models and Chan-Paton charges [4], to confine our attention to the open descendants of Rational Conformal Field Theory [5, 6, 0, 8, 9, 10]. These extend Rational Conformal Field Theory [1] to surfaces with boundaries [12, 13, 14] and/or crosscaps, and exhibit neatly many interesting features. The resulting presentation should complement refs. [15]. The following four sections, devoted to the four amplitudes that define the spectrum, include two instructive examples drawn from our previous study [9] of the $A D E$ series [16] of $S U(2)$ WZW models [17]. In Section 2 we review the structure of the oriented closed sector. This involves the torus amplitude, by now standard knowledge, but sets the stage for the construction of open descendants and allows us to introduce our notation and conventions. In Section 3 we discuss the Klein-bottle projection of the closed spectrum. In Section 4 we discuss the annulus amplitude and the related issue of boundary operators, including our recent work on boundary operators in non-diagonal models [10]. This part should also be of some interest in Condensed Matter Physics, where boundary operators can describe the effects of impurities near criticality [18]. Finally, in Section 5 we discuss the Möbius-strip projection of the open spectrum. 


\section{The Torus Amplitude}
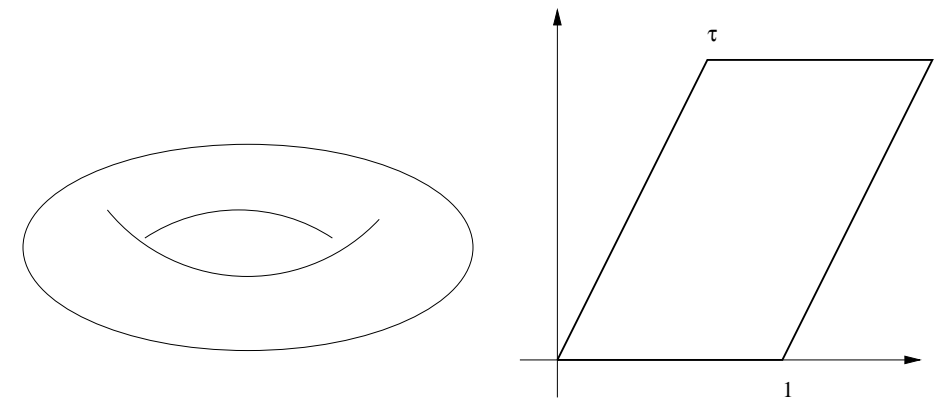

Figure 1. The torus amplitude

The first amplitude of our construction is the torus of fig. 1. Its fundamental polygon, a parallelogram, has one vertex at the origin and two sides ending at 1 and $\tau$, with $\operatorname{Im}(\tau)>0$. We restrict our attention to a two-dimensional rational conformal field theory with a symmetry algebra $\mathcal{A} \times \overline{\mathcal{A}}$, such that

- the left and right symmetry algebras are identical;

- the symmetry algebra is maximally extended.

The two assumptions imply a one-to-one correspondence between the two-dimensional field content and the chiral content, described by a finite set of primary fields $\left\{\varphi_{i}\right\}$ of $\mathcal{A}$. These can be neatly represented by the (Virasoro reduced) characters of the symmetry algebra

$$
\chi_{i}(\tau)=\operatorname{Tr}_{\mathcal{H}_{i}} e^{2 \pi i \tau \hat{L}_{0}}
$$

where the trace is over the corresponding $\mathcal{A}$ module. Since the left and right symmetry algebras coincide, the same is true for the corresponding sets of characters, as well as for the primary fields. The left-right symmetry is a necessary condition to construct open descendants. On the other hand, the assumption of maximality can well be relaxed, although it simplifies considerably the resulting formulas. For instance, the $D_{\text {even }}$ models may be treated as $s u(2)$ models or as extended symmetry ones.

The two generators of the $P S L(2, Z)$ group of modular transformations act linearly 
on the characters, according to

$$
\chi_{i}\left(-\frac{1}{\tau}\right)=\sum_{j} S_{i j} \chi_{j}(\tau) \quad \chi_{i}(\tau+1)=\sum_{j} T_{i j} \chi_{j}(\tau)
$$

where $S$ and $T$ are unitary symmetric matrices that satisfy $S^{2}=(S T)^{3}=C$, with $C$ the conjugation matrix and $C^{2}=1$. All the infinitely many choices of fundamental cell in the lattice generated by the parallelogram in fig. 1 may be related by the two transformations of eq. (2.2). Modular invariance thus makes the choice of fundamental cell immaterial. The matrix $T$, diagonal in the basis of characters, encodes the conformal weights of the primary fields.

The two-dimensional field content can be read from the modular invariant partition function

$$
Z_{T}=\sum_{i, j} \chi_{i} X_{i j} \chi_{j} \quad S X S^{\dagger}=X \quad T X T^{\dagger}=X
$$

where the non-negative integers $X_{i j}$ count the multiplicities of the two-dimensional fields $\varphi_{i, \bar{j}}(z, \bar{z})$ in the bulk spectrum. The assumption that the symmetry algebra $\mathcal{A}$ be maximal implies that the torus partition function, if not diagonal, is at most a permutation invariant. Thus, we are effectively restricting our attention to models with

$$
X_{i j}=\delta_{i \sigma(j)}
$$

where $\sigma(j)$ denotes a permutation of the labels $j$. Each chiral character is always coupled to a single antichiral one, and there are no multiplicities larger than one in the bulk spectrum.

Denoting by $[\varphi]$ the conformal family of $\varphi$, one has the fusion algebra

$$
\left[\varphi_{i}\right] \times\left[\varphi_{j}\right]=\sum_{k} N_{i j}^{k}\left[\varphi_{k}\right]
$$

where the Verlinde formula [19]

$$
N_{i j}^{k}=\sum_{\ell} \frac{S_{i \ell} S_{j \ell} S_{k \ell}^{\dagger}}{S_{1 \ell}}
$$

relates the fusion-rule coefficients $N_{i j}{ }^{k}$ to the modular matrix $S$. The integers $N_{i j}{ }^{k}$ encode the basic information in the OPE [21] of any pair of fields. 
It should be appreciated that modular invariance plays also the role of a completeness condition for two-dimensional fields. Any set of fields whose torus amplitude is not modular invariant, even if closed under the operator product, should be consistently enlarged by the inclusion of other fields. In Section 4 we shall see that extending the completeness requirement to the open sector reduces the classification problem of the allowed boundary states to a set of polynomial equations closely related to the Verlinde algebra [10].

We shall illustrate the construction for the $k=2\left(A_{3}\right)$ and for the $k=6\left(D_{5}\right)$ su(2) WZW models, the simplest ones in the $A$ and $D_{\text {odd }}$ series. General formulae valid for the whole series, as well as the treatment of $D_{\text {even }}$ and $E$ models, may be found in refs. [9, 10]. We label the characters by shifted $s u(2)$ weights, related to the isospin by $i=2 I+1$. In this notation the corresponding modular invariants are:

- for the $A_{3}$ model

$$
Z_{T}^{A_{3}}=\left|\chi_{1}\right|^{2}+\left|\chi_{2}\right|^{2}+\left|\chi_{3}\right|^{2}
$$

- for the $D_{5}$ model

$$
Z_{T}^{D_{5}}=\left|\chi_{1}\right|^{2}+\left|\chi_{3}\right|^{2}+\left|\chi_{5}\right|^{2}+\left|\chi_{7}\right|^{2}+\left|\chi_{4}\right|^{2}+\chi_{2} \bar{\chi}_{6}+\chi_{6} \bar{\chi}_{2}
$$

\section{The Klein-Bottle Amplitude}
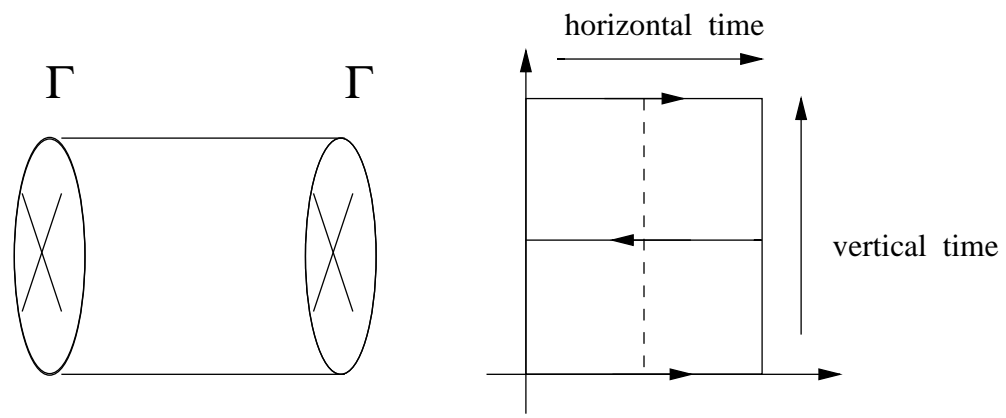

Figure 2. The Klein-bottle amplitude

The construction of open descendants involves surfaces with boundaries and crosscaps. The crosscap, the simplest non-orientable surface, may be pictured as a disk with dia- 
metrically opposite points identified. The Klein bottle is a closed non-orientable surface with two crosscaps and no handles.

The introduction of crosscaps breaks the $\mathcal{A} \times \overline{\mathcal{A}}$ symmetry of the 2-dimensional model. Here we confine our attention to crosscaps preserved by a residual $\mathcal{A}$ symmetry, and in Section 4 we shall extend this requirement to the boundaries. There are, however, two crucial differences between the crosscap and the more familiar case of a boundary : the involution that defines the crosscap has no fixed points, and the crosscap itself may not be localized.

The crosscap interchanges chiral and antichiral parts of two-dimensional fields, and thus defines an involution

$$
\Omega: \varphi_{i \bar{i}} \rightarrow \epsilon_{i} \varphi_{\bar{i} i} \quad, \quad \epsilon_{i}=\epsilon_{\bar{i}}= \pm 1
$$

Since we have a one-to-one correspondence between two-dimensional and chiral spectra, the action of this involution can be extended to the chiral characters. Consistency with the fusion rules demands that the signs $\epsilon_{i}$ satisfy the constraints

$$
\text { if } \quad N_{i j k} \neq 0 \quad \text { then } \quad \epsilon_{i} \epsilon_{j} \epsilon_{k}=1 \text {. }
$$

The Klein bottle is pictured in figure 2, together with the corresponding polygon, that in this case is a rectangle, so that the Teichmüller parameter $\tau$ is purely imaginary. The two vertical sides are directly identified, while the two horizontal ones are identified after an orientation reversal. There are two canonical inequivalent choices of time, and consequently the ultraviolet point $\tau=0$ is not excluded form the moduli space. Referring to fig. 2, "vertical" time exhibits the propagation of closed strings undergoing a turnover, and one has

$$
K=\sum_{i} \chi_{i} K^{i}
$$

The $K^{i}$, integers constrained to satisfy $\left|K^{i}\right|=X_{i i}$, are always 0 or \pm 1 for a permutation invariant. They are related to the $\epsilon_{i}$, and should also satisfy eqs. (3.2).

A modular $S$ transformation to "horizontal" time turns eq. (3.3) into the transverse (vacuum) channel that describes the propagation of closed strings on a tube terminating 
at the two crosscaps,

$$
\tilde{K}=\sum_{i} \chi_{i} \Gamma_{i}^{2}
$$

The reflection coefficients $\Gamma_{i}$ determine the one-point functions of the bulk fields in front of the crosscap.

In fig. 2 the two crosscaps may be associated with the left border of the rectangle and with the dashed line. The transverse channel may then be identified with the portion of the double cover limited by these two lines.

The n-point functions of the two-dimensional fields in front of a crosscap can be expressed in terms of the corresponding 2n-point chiral conformal blocks. In particular, the $\Gamma_{i}$ can be non-vanishing only for fields whose left and right conformal dimensions coincide, $\left(\Delta_{i}=\Delta_{\bar{i}}\right)$, and only if $\epsilon_{i}=1$. The consistency of two-point functions with the involution of eq. (3.1) implies the "crosscap constraint" 8, 9], a set of linear relations for the $\Gamma_{i}$ and the signs $\epsilon_{j}$ involving also the two-dimensional structure constants and the fusion matrix $F$. In general, these determine completely the $\Gamma_{i}$, and thus, after an $S$ modular transformation, the direct-channel coefficients $K^{i}$. Although it is usually simpler to solve directly the system of eqs. (3.2) for $K^{i}$, this does not determine the signs of the $\Gamma_{i}$ that, as we shall see, are needed in the Möbius-strip amplitude.

In the projected spectrum, $\frac{1}{2}\left(Z_{T}+K\right)$, the Verma modules are (anti)symmetrized under left-right interchange. Multiple solutions, whenever present, describe inequivalent projections of the closed spectrum, and the complete open descendants have correspondingly different spectra.

There are two possible choices for the Klein bottle partition functions both in the $A_{3}$ and in the $D_{5}$ models. Anticipating the structure of the resulting Chan-Paton groups, we shall denote them by (r)eal and (c)omplex respectively. The direct channel expressions corresponding to the modular invariants of eqs. (2.7) and (2.8) are then

$$
\begin{aligned}
& K_{r}^{A_{3}}=\chi_{1}-\chi_{2}+\chi_{3}, \\
& K_{c}^{A_{3}}=\chi_{1}+\chi_{2}+\chi_{3}, \\
& K_{r}^{D_{5}}=\chi_{1}+\chi_{3}+\chi_{5}+\chi_{7}-\chi_{4},
\end{aligned}
$$




$$
K_{c}^{D_{5}}=\chi_{1}+\chi_{3}+\chi_{5}+\chi_{7}+\chi_{4}
$$

It is instructive to compare these expressions with the signs $\epsilon_{i}$ of eq. (3.1), that in both models are

- $\epsilon_{i}=1$ for all $i$ in the real case;

$-\epsilon_{i}=(-1)^{i-1}$ in the complex case.

The relative factor $(-1)^{2 I}$ between $K_{i}$ and $\epsilon_{i}$ is introduced by the $s u(2)$ structure of the fields. It should be appreciated that, differently from the $K_{i}$, the $\epsilon_{i}$ are well defined for all fields.

\section{The Annulus Amplitude}

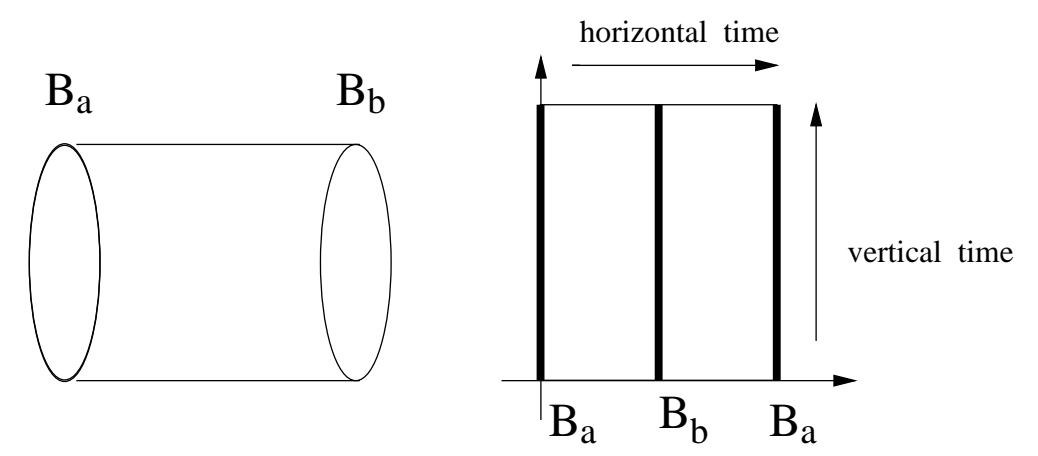

Figure 3. The annulus amplitude

Let us now turn to the annulus amplitude of fig. 3, whose fundamental polygon is again a rectangle. The horizontal sides identified, while the vertical sides represent the two boundaries. The main issue of this Section is to indicate how to construct, for each given model, the allowed types of boundary states.

The annulus is doubly covered by a torus whose fundamental polygon is also a rectangle, and therefore there is again only one real Teichmüller parameter. The two canonical choices of "time", that referring to fig. 3 may be termed "horizontal" and "vertical", have quite distinct roles, and consequently the range of the parameter is again the whole positive imaginary axis. As in the Klein-bottle amplitude, the ultraviolet point $\tau=0$ 
is not excluded from the modular region. The two choices of time have quite distinct roles: vertical time exhibits a vacuum amplitude for open strings, while horizontal time exhibits an amplitude for closed strings propagating between two boundaries. Therefore, this diagram gives information on the boundary operators, a new sector of the operator space, but at the same time it rests on the known spectrum of the closed model. This crucial property, discovered very early in the bosonic string, played a central role in the proposal of ref. [5]. Indeed, the annulus amplitude determines to a large extent the open sector of the theory in terms of its closed sector. The other two surfaces, the Klein bottle and the Möbius strip, lead to additional, related truncations of closed and open spectra.

The introduction of boundaries breaks the total $\mathcal{A} \times \overline{\mathcal{A}}$ symmetry of the two-dimensional model, and can actually do so to different extents, leading to different classes of descendants. The restriction to conformally invariant boundaries is natural in String Theory. As in Section 3, we actually confine our attention to the maximal case, where the residual symmetry is $\mathcal{A}$. Only one Virasoro algebra acts on the fields in this case, with the same central charge as both the chiral and antichiral ones. Hence, both the representations and the characters of the bulk and boundary algebras coincide. This implies that the n-point functions of two-dimensional fields solve the same differential equations as the 2n-point chiral conformal blocks, while the corresponding partition function is linear (rather than sesquilinear) in the same characters.

Following Cardy [12], one can introduce boundary conditions corresponding to different boundary states of the residual $\mathcal{A}$ symmetry, as well as boundary operators $\psi_{i}^{a b}$ that mediate between them. On the annulus one can define two different partition functions. The direct channel (corresponding to the "vertical" time in fig. 3) exhibits the propagation of open strings with ends on the two boundaries, and

$$
A=\sum_{i, a, b} \chi_{i} A_{a b}^{i} n^{a} n^{b}
$$

where the non-negative integers $A_{a b}^{i}$ count the multiplicities of the boundary operators $\psi_{i}^{a b}$ in the spectrum. We have allowed for multiplicities $n^{a}$ associated with the boundaries, and the boundary operators can thus be matrix valued. In open-string models this extension 
results in the introduction of Chan-Paton groups, restricted by the factorization of treelevel amplitudes to the three infinite families $U(n), O(n)$ and $U S p(2 n)$ | 1 . The boundaries are valued in the fundamental representations of these classical Lie groups, and one is to distinguish two cases. When the group is $U(n)$, there are two inequivalent choices for the fundamental representation, that can thus be associated with oriented boundaries. On the other hand, no orientation is needed for $U S p(2 n)$ and $O(2 n)$, whose fundamental representations are (pseudo)real. Alternatively, in applications to Statistical Mechanics, one may regard eq. (4.1) as a formal polynomial in the variables $n^{a}$, a generating function for the multiplicities of the allowed boundary fields.

The transverse or vacuum channel (corresponding to the "horizontal" time in fig. 3) has a very different interpretation: it exhibits the propagation of closed strings along a tube terminating at the two boundaries. Since the closed spectrum undergoes a pair of reflections, this amplitude is linear in the characters, with coefficients that are perfect squares

$$
\tilde{A}=\sum_{i} \chi^{i}\left[\sum_{a} B_{i a} n^{a}\right]^{2}
$$

$B_{i a}$, the reflection coefficient for the sector $i$ of the bulk spectrum from a boundary of type $a$, is proportional to the corresponding disk one-point function. The sum within brackets is the total reflection coefficient for the sector $i$.

Since $\tilde{A}$ is related to $A$ by a modular $S$ transformation, the open spectrum is closely linked to the disk one-point functions. Cardy's ansatz [12] determines the open spectrum when the matrix $X$ in eq. $(2.3)$ is the conjugation matrix $C$. In this case both the open sectors and the types of boundaries are in one-to-one correspondence with the bulk spectrum, $A_{i j}^{k}=N_{i j}^{k}$, and thus the fusion-rule coefficients count the available boundary operators.

In the presence of boundaries, one can introduce two new types of operator products [13, 14. The first,

$$
\psi_{i}^{a b} \psi_{j}^{b c}=\sum_{\ell} C_{i j \ell}^{a b c} \psi_{\ell}^{a c}
$$

involves boundary fields only and plays a central role in the definition of their disk am- 
plitudes. The second,

$$
\left.\varphi_{i \bar{i}}\right|_{a}=\sum_{\ell} C_{(i \bar{i}) \ell}^{a} \psi_{\ell}^{a a},
$$

involves both bulk and boundary fields, and describes the behavior of bulk fields in front of the different boundaries. The $B_{i a}$ in eq. (4.2) are proportional to the $C_{(i \bar{i}) 1}^{a}$, with coefficients that depend on the normalizations of the bulk and boundary fields. The new structure constants, $C_{i j \ell}^{a b c}$ and $C_{(i \bar{i}) \ell}^{a}$, and thus the reflection coefficients in $\tilde{A}$, can all be determined by imposing the consistency of the full set of OPE's. This leads to new sewing constraints, introduced in [14]. Some errors in the final expressions, related to the analytic continuations involved, were corrected in [10], using also ref. [20]. These constraints extend the original ones of refs. [21, 22] (the non-planar duality of bulk fourpoint amplitudes and the modular invariance of torus one-point amplitudes), and lead to (quadratic or cubic) relations for the structure constants. The method is quite powerful and gives very detailed information on the theory, but it requires explicit expressions for the braid and fusion matrices of the chiral conformal blocks. These, however, are known only in a limited number of cases. This situation can be compared to a similar one for the oriented closed sector. Modular invariant partition functions are classified for vast classes of models (see e.g. [23] and references therein), while the two-dimensional structure constants are completely known only in a few cases (essentially for some abelian models and for minimal and $S U(2)$ WZW models).

This suggests an approach to the problem of classifying boundary states similar, in some respects, to the one followed for closed orientable models. Indeed, the integer coefficients $A_{a b}^{i}$ in the direct-channel annulus amplitude of eq. (4.1) have a nice interpretation as multiplicities of the corresponding boundary operators. Moreover, our two assumptions on the structure of the symmetry algebra imply that they also count the different couplings $\left\langle a\left|\varphi_{i \bar{i}}\right| b\right\rangle$, since there is a one-to-one correspondence between two-dimensional and chiral fields. For a complete set of boundary states (we have already stressed that modular invariance plays a similar role for the closed sector), one can derive the following set of polynomial equations [10] involving also the fusion-rule coefficients $N_{i j}^{k}$

$$
\sum_{b} A_{a}^{i b} A_{b c}^{j}=\sum_{k} N_{k}^{i j} A_{a c}^{k},
$$




$$
\sum_{i} A_{i a b} A_{c d}^{i}=\sum_{i} A_{i a c} A_{b d}^{i} .
$$

Upper and lower boundary indices are to be distinguished whenever complex charges (corresponding to oriented boundaries) are present. Moreover, the matrix $\left(A_{1}\right)_{a b}=\left(A_{1}\right)^{a b}$ is the metric for the boundary indices, since it follows from eq. (4.5) that $\sum_{b} A_{i a b} A_{1}^{b c}=$ $A_{i a}^{c}$, while $\left(A_{1}\right)_{a}^{b}=\delta_{a}^{b}$.

Eqs. (4.5) and (4.6) do not determine completely the matrices $A_{i}^{a b}$, since they contain only chiral data. The torus modular invariant of eq. (2.3) is another crucial ingredient. Indeed, if for some $j$ the matrix element $X_{j \bar{j}}$ of the modular invariant vanishes, the tube can not support the corresponding mode, and the bulk-to-boundary structure constants $C_{(j \bar{j}) 1}^{a}$ (and thus the reflection coefficients $B_{j a}$ ) also vanish for all values of $a$. After a modular $S$ transformation this implies that, for all $a$ and $b$,

$$
\sum_{i} A_{i a b} S_{j}^{i}=0
$$

Hence, the $A_{i a b}$ are in general linearly dependent matrices, while the number of different boundary states can be expressed rather neatly in terms of the corresponding modular invariant as $\operatorname{Tr}(X C)$. Eqs. (4.5)-(4.7) have in general multiple solutions, but in all cases that we have analyzed explicitly they determine $A_{i a b}$ up to the orientation of (pairs of) boundaries. The solution corresponding to a given $\left(A_{1}\right)_{a b}$ is unique, while different choices for $\left(A_{1}\right)_{a b}$ are related by the action of a simple current that squares to the identity.

As an illustration, we shall again resort to the $A_{3}$ and $D_{5} s u(2)$ WZW models. Since the $A_{3}$ model is diagonal, in the real case $A_{i j k}=N_{i j k}$, and

$$
A_{r}^{A_{3}}=\chi_{1}\left(n_{1}^{2}+n_{2}^{2}+n_{3}^{2}\right)+\chi_{2}\left(2 n_{1} n_{2}+2 n_{2} n_{3}\right)+\chi_{3}\left(2 n_{1} n_{3}+n_{2}^{2}\right) .
$$

The second solution may be obtained acting on this spectrum with the simple current that corresponds to $\chi_{3}$. It has a pair of complex charges that we denote by $n$ and $\bar{n}$, and

$$
A_{c}^{A_{3}}=\chi_{1}\left(n_{2}^{2}+2 n \bar{n}\right)+\chi_{2}\left(2 n_{2} n+2 n_{2} \bar{n}\right)+\chi_{3}\left(n^{2}+\bar{n}^{2}+n_{2}^{2}\right) .
$$

The equality $\bar{n}=n$ ensures the positivity of the transverse-channel amplitude.

In the $D_{5}$ case the expressions are more complicated, and therefore a few remarks are in order. Since $\chi_{2}$ and $\chi_{6}$ enter off-diagonally the torus modular invariant (2.8), eqs. (4.7) 
limit to five the possible types of charges. Hence, the algebra of the boundary operators is different from the (chiral) bulk algebra, that involves seven types of primary fields. In particular, there are boundary operators with multiplicities larger than one (some of the terms involving the $n_{5}$ charge below). As discussed in ref. [10], this corresponds to a new phenomenon, whereby the boundary algebra is extended by a simple current, in this case of dimension $\frac{3}{2}$, that can not extend the bulk algebra. The real assignment corresponding to $\left(A_{1}\right)_{a b}=\delta_{a b}$ is

$$
\begin{aligned}
& A_{r}^{D_{5}}=\chi_{1}\left(n_{1}^{2}+n_{2}^{2}+n_{3}^{2}+n_{4}^{2}+n_{5}^{2}\right)+\left(\chi_{2}+\chi_{6}\right)\left(2 n_{1} n_{2}+2 n_{1} n_{5}+2 n_{3} n_{5}+2 n_{4} n_{5}\right)+ \\
& \quad \chi_{3}\left(n_{1}^{2}+2 n_{1} n_{3}+2 n_{1} n_{4}+2 n_{3} n_{4}+2 n_{2} n_{5}+2 n_{5}^{2}\right)+ \\
& \quad \chi_{4}\left(4 n_{1} n_{5}+2 n_{2} n_{3}+2 n_{3} n_{5}+2 n_{2} n_{4}+2 n_{4} n_{5}\right)+ \\
& \quad \chi_{5}\left(n_{1}^{2}+n_{3}^{2}+n_{4}^{2}+2 n_{5}^{2}+2 n_{1} n_{3}+2 n_{1} n_{4}+2 n_{2} n_{5}\right)+\chi_{7}\left(n_{1}^{2}+n_{2}^{2}+n_{5}^{2}+2 n_{3} n_{4}\right) .
\end{aligned}
$$

The complex assignment has only one complex pair of charges $\bar{n}=n$ (replacing the $n_{3}$ and $n_{4}$ charges), and is obtained from the real one acting with the simple current in $\chi_{7}$

$$
\begin{aligned}
& A_{c}^{D_{5}}=\chi_{1}\left(n_{1}^{2}+n_{2}^{2}+2 n \bar{n}+n_{5}^{2}\right)+\left(\chi_{2}+\chi_{6}\right)\left(2 n_{1} n_{2}+2 n_{1} n_{5}+2 n n_{5}+2 \bar{n} n_{5}\right)+ \\
& \chi_{3}\left(n_{1}^{2}+n^{2}+\bar{n}^{2}+2 n_{1} n+2 n_{1} \bar{n}+2 n_{2} n_{5}+2 n_{5}^{2}\right)+ \\
& \quad \chi_{4}\left(4 n_{1} n_{5}+2 n_{2} n+2 n_{2} \bar{n}+2 n n_{5}+2 \bar{n} n_{5}\right)+ \\
& \quad \chi_{5}\left(n_{1}^{2}+2 n_{5}^{2}+2 n_{1} n+2 n_{1} \bar{n}+2 n_{2} n_{5}+2 n \bar{n}\right)+\chi_{7}\left(n_{1}^{2}+n_{2}^{2}+n_{5}^{2}+n^{2}+\bar{n}^{2}\right)
\end{aligned}
$$

More details on the explicit solution of this model may be found in ref. [10.

\section{The Möbius-Strip Amplitude}

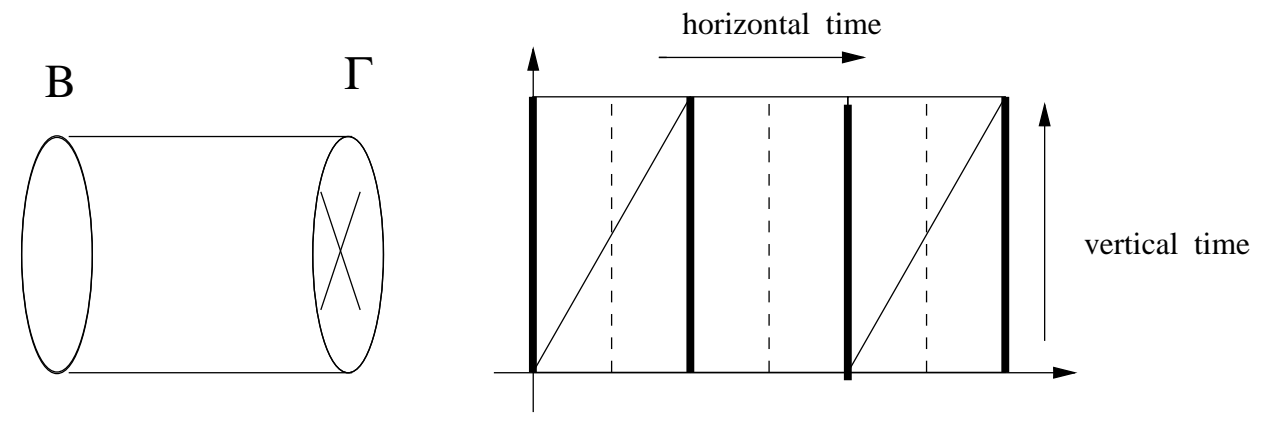

Figure 4. The Moebius-strip amplitude 
The Möbius-strip amplitude in fig. 4 is the last surface in our construction of the spectrum. Though perhaps more familiar than the Klein bottle, this surface has the peculiar feature of having a double cover with a non-vanishing real part of $\tau$. Its value, $1 / 2$, induced by the relative orientation of the horizontal sides, has an important effect: the contributions of a given Verma module have in this case alternating signs that depend on the level. The Möbius strip can be viewed as a tube terminating at one boundary and one crosscap. The crosscap may be associated with the dashed line in fig. 4, whose points are all identified in pairs in the double cover. On the other hand, the boundary comprises the two vertical sides of the rectangle. Even in this case there are two canonical but inequivalent choices of time. "Vertical" time exhibits the propagation of open strings undergoing a "flip" in their orientation, while "horizontal" time exhibits the propagation of closed strings between a boundary and a crosscap. The amplitude corresponding to the latter choice, determined by the boundary and crosscap reflection coefficients $B_{i a}$ and $\Gamma_{i}$, is thus

$$
\tilde{M}=\sum_{i} \hat{\chi}_{i} \Gamma_{i}\left[\sum_{a} B_{i a} n^{a}\right] .
$$

In order to give a meaning to this equation, one has to make corresponding choices for the reflection coefficients $B_{i a}$ and $\Gamma_{i}$. These are determined comparing $\tilde{M}$ (after a $P$ transformation, to be defined shortly) to the direct channel amplitude

$$
M=\sum_{i} \hat{\chi}_{i} M_{a}^{i} n^{a}
$$

and thus by consistency with the direct-channel annulus amplitude of eq. (4.1). The coefficients $M_{a}^{i}$ can be interpreted as twists of the open-string spectrum, and must be integers satisfying

$$
M_{a}^{i}=A_{a a}^{i} \quad(\bmod 2)
$$

a relation slightly more general than the one between direct Klein bottle and torus coefficients, since boundary operators can have multiplicities $A_{a a}^{i}$ greater than one. Imposing eqs. (5.3) singles out pairs of compatible annulus and Klein bottle projections and completes the construction of the open descendants.

Let us return to the relation between the two forms of the Möbius amplitude. As we have seen, for the direct channel the natural modular parameter is $(i \tau+1) / 2$. On the 
other hand, for the vacuum channel it is $(i+\tau) / 2 \tau$. Due to the non-vanishing real part of $\tau$, it is convenient to work in the basis of real characters

$$
\hat{\chi}_{j}=e^{-i \pi\left(\Delta_{j}-c / 24\right)} \chi_{j}\left(\frac{i \tau+1}{2}\right)
$$

The modular transformation that links direct and transverse channels is then given by the matrix

$$
P=T^{1 / 2} S T^{2} S T^{1 / 2}
$$

that, just like $S$, satisfies $P^{2}=C$.

By a relation reminiscent of eq. (2.6), from $S$ and $P$ one can construct the integervalued matrices [9]

$$
\left(Y_{i}\right)_{j}^{k}=\sum_{\ell} \frac{S_{i \ell} P_{j \ell} P_{k \ell}^{\dagger}}{S_{1 \ell}},
$$

that form an abelian algebra and satisfy the relations

$$
\left(Y_{i}\right) \cdot\left(Y_{j}\right)=\sum_{\ell} N_{i j}^{\ell}\left(Y_{\ell}\right)
$$

One can then write compact expressions for the Möbius partition function (5.2) in terms of $Y_{i j k}$. In particular, if the torus modular invariant is built with the conjugation matrix $C$, the boundary and (chiral) bulk spectra coincide and, as we have seen, the annulus multiplicities are equal to the fusion rule coefficients $N_{i j k}$. One solution for the twists in eq. (5.2) is then $M_{i j}=Y_{j i 1}$, while the direct-channel Klein-bottle coefficients are in this case $K^{i}=Y_{i 11}$. Similar representations hold for other descendants of the same modular invariant. The situation becomes more intricate whenever the boundary algebra is substantially different from the chiral one. An important instance of this phenomenon, the non-diagonal $D_{\text {odd }} s u(2)$ WZW models, suggest that one can always embed the open spectrum in an auxiliary diagonal model.

In the complete partition function of the open sector, $\frac{1}{2}(A+M)$, the signs of the $n_{a}$ terms in $M$ determine the symmetry of the states under the interchange of the two (equal) Chan-Paton charges at the ends. Note that one can change the overall sign of the Möbius amplitude by replacing all $n_{a}$ with $-n_{a}$. In open-string models the signs, as well as the 
sizes of the Chan-Paton groups, are further constrained by tadpole conditions, while the functional measure introduces an additional factor of two in $\tilde{M}$.

Returning to our examples, the Möbius amplitudes for the $A_{3}$ model are standard and can be directly expressed in terms of $Y_{i j k}$

$$
\begin{aligned}
& M_{r}^{A_{3}}=\hat{\chi}_{1}\left(n_{1}-n_{2}+n_{3}\right)+\hat{\chi}_{3}\left(n_{2}\right) \\
& M_{c}^{A_{3}}=\hat{\chi}_{1}\left(n_{2}\right)+\hat{\chi}_{3}\left(n+\bar{n}+n_{2}\right) .
\end{aligned}
$$

On the other hand, the $D_{5}$ model exhibits non-trivial solutions of eq. (5.3), since the $2 n_{5}^{2}$ in the annulus amplitudes of eqs. (4.10) and (4.11) can correspond either to a $2 n_{5}$ or to a zero Möbius contribution. In the first case the two sets of states have the same twist, while in the second case their twists are opposite. The expressions for the two types of descendants are

$$
\begin{gathered}
M_{r}^{D_{5}}=\hat{\chi}_{1}\left(n_{1}-n_{2}+n_{3}+n_{4}-n_{5}\right)+\hat{\chi}_{3}\left(-n_{1}+2 n_{5}\right)+ \\
\hat{\chi}_{5}\left(n_{1}+n_{3}+n_{4}\right)+\hat{\chi}_{7}\left(n_{1}+n_{2}+n_{5}\right),
\end{gathered}
$$

and

$$
\begin{aligned}
M_{c}^{D_{5}}=\hat{\chi}_{1}\left(-n_{1}+n_{2}+n_{5}\right)+\hat{\chi}_{3}\left(n_{1}+n+\bar{n}\right)+ \\
\hat{\chi}_{5}\left(n_{1}+2 n_{5}\right)+\hat{\chi}_{7}\left(n_{1}+n_{2}+n+\bar{n}+n_{5}\right) .
\end{aligned}
$$

\section{Acknowledgments}

We are grateful to the Organizers of the CERN Meeting on STU Dualities and to the Organizers of the Rome Triangle Meeting for their kind invitations. We are also grateful to Massimo Bianchi and Gianfranco Pradisi for an enjoyable collaboration on several aspects

of this problem. This work was supported in part by E.E.C. Grant CHRX-CT93-0340. 


\section{References}

[1] J. Polchinski, Phys. Rev. Lett. 75 (1995) 4724;

E. Witten, hep-th 9510135, 9511030;

J. Polchinski and E. Witten, hep-th 9510169;

P. Horava and E. Witten, hep-th 9510209, 9603142;

C. Bachas, hep-th 9511043;

E.G. Gimon and J. Polchinski, hep-th 9601038;

A. Dabolkhar and J. Park, hep-th 9602030, 9604178;

E.G. Gimon and C.V. Johnson, hep-th 9604129.

[2] An incomplete list of references is:

G. Veneziano, Nuovo Cimento 57A (1968) 190;

V. Alessandrini, Nuovo Cimento 2A (1971) 321;

V. Alessandrini and D. Amati, Nuovo Cimento 4A (1971) 222;

E. Cremmer and J. Scherk, Nucl. Phys. B50 (1972) 509;

L. Clavelli and J. Shapiro, Nucl. Phys. B57 (1973) 490;

A. Chodos and C. Thorn, Nucl. Phys. B54 (1974) 509;

E. Corrigan and D. Fairlie, Nucl. Phys. B91 (1975) 545;

W. Siegel, Nucl. Phys. B109 (1976) 244;

G. Venturi, Nuovo Cimento 16A (1976) 484;

M.B. Green and J.H. Schwarz, Phys. Lett. B149 (1984) 117, B151 (1985) 21;

M.R. Douglas and B. Grinstein, Phys. Lett. B183 (1987) 52;

S. Weinberg, Phys. Lett. B183 (1987) 287;

J.A. Harvey and J.A. Minahan, Phys. Lett. B188 (1987) 44;

S.K. Blau, M. Clements, S. Della Pietra, S. Carlip, V. Della Pietra,

Nucl. Phys. B301 (1988) 285;

M. Bianchi and A. Sagnotti, Phys. Lett. B211 (1988) 407;

C.G. Callan, C. Lovelace, C. Nappi and S.A. Yost, Nucl. Phys. B308 (1988) 221;

J. Polchinski, Phys. Lett. B203 (1988) 39;

J. Polchinski and Y. Cai, Nucl. Phys. B296 (1988) 91; 
L. Clavelli, P. Elmfors, P.H. Cox, Phys. Rev. D40 (1989) 4078;

G. Pradisi and A. Sagnotti, Phys. Lett. B216 (1989) 59;

J. Gowaerts, Phys. Lett. B220 (1989) 77;

P. Horava, Nucl. Phys. B327 (1989) 461, Phys. Lett. B231 (1989) 251;

N. Ishibashi and T. Onogi, Nucl. Phys. B318 (1989) 239;

N. Ishibashi, Mod. Phys. Lett. A4 (1989) 251;

Z. Bern and D.C. Dumbar, Nucl. Phys. B319 (1989) 104;

J. Dai, R.G. Leigh and J. Polchinski, Mod. Phys. Lett. A4 (1989) 2073;

P. Horava, hep-th 9404101;

M.B. Green, Phys. Lett. B266 (1991) 325;

A. Sagnotti, Phys. Lett. B294 (1992) 196;

M.B. Green and J. Polchinski, Phys. Lett. B335 (1994) 377;

additional references may be found in:

M.B. Green, J.H. Schwarz and E. Witten, "Superstring Theory" (Cambridge, 1987);

J.H. Schwarz, ed., "Superstring Theory" (World Scientific, 1985);

M. Jacob, ed, "Dual Theory" (North-Holland, 1974).

[3] P.K. Townsend, hep-th 9512062;

E. Witten, hep-th 9512219.

[4] J.E. Paton and H.M. Chan, Nucl. Phys. B10 (1969) 516;

J.H. Schwarz, in "Current Problems in Particle Theory",

Proc. J. Hopkins Conf. 6 (Florence, 1982);

N. Marcus and A. Sagnotti, Phys. Lett. B119 (1982) 97, B188 (1987) 58.

[5] A. Sagnotti, in "Non-Perturbative Quantum Field Theory", eds. G. Mack et al (Pergamon Press, 1988), p. 521.

[6] M. Bianchi and A. Sagnotti, Phys. Lett. B247 (1990) 517, Nucl. Phys. B361 (1991) 519.

[7] M. Bianchi, G. Pradisi and A. Sagnotti, Phys. Lett. B273 (1991) 389, Nucl. Phys. B376 (1992) 365.

[8] D. Fioravanti, G. Pradisi and A. Sagnotti, Phys. Lett. B321 (1994) 349. 
[9] G. Pradisi, A. Sagnotti and Ya.S. Stanev, Phys. Lett. B354 (1995) 279, B356 (1995) 230.

[10] G. Pradisi, A. Sagnotti and Ya.S. Stanev, hep-th 9603097.

[11] G. Moore and N. Seiberg, Phys. Lett. B212 (1988) 451, Nucl. Phys. B313 (1989) 16.

[12] J.L. Cardy, Nucl. Phys. B324 (1989) 581.

[13] J.L. Cardy and D.C. Lewellen, Phys. Lett. B259 (1991) 274.

[14] D.C. Lewellen, Nucl. Phys. B372 (1992) 654.

[15] A. Sagnotti, hep-th 9509080;

G. Pradisi, hep-th 9603104.

[16] A. Cappelli, C. Itzykson and J.B. Zuber, Comm. Math. Phys. 113 (1987) 1.

[17] E. Witten, Comm. Math. Phys. 92 (1984) 455;

V.G. Knizhnik, A.B. Zamolodchikov, Nucl. Phys. B247 (1984) 83.

[18] A.W.W. Ludwig, Int. J. Mod. Phys. B8 (1994) 347;

I. Affleck, cond-mat 9512099, and references therein.

[19] E. Verlinde, Nucl. Phys. B300 (1988) 360.

[20] Ya.S.Stanev, I.T.Todorov, L.K.Hadjiivanov, Phys. Lett. B276 (1992) 87.

[21] A.A. Belavin, A.M. Polyakov and A.B. Zamolodchikov, Nucl. Phys. B241 (1984) 333.

[22] H. Sonoda, Nucl. Phys. B311 (1988) 401.

[23] T.Gannon, hep-th 9408119. 